\title{
Creating Psychological Safety on Medical Teams in Times of Crisis
}

\author{
Lily Rotman Devaraj, MD ${ }^{1,2 \star}$, Cynthia Cooper, MD ${ }^{1,2}$, Arabella Simpkin Begin, MD, MMSc ${ }^{1,2,3}$
}

'Department of Medicine, Massachusetts General Hospital, Boston, Massachusetts; ${ }^{2}$ Harvard Medical School, Boston, Massachusetts; ${ }^{3}$ Department of Pharmacology, University of Oxford, Oxford, United Kingdom.

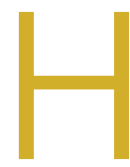

ospitalized patients receive care via a team-based approach. Because of frequent turnover and constant changes in team members, medical teams require rapid establishment of psychological safety. Psychological safety, or "being able to show and employ one's self without fear of negative consequences of self-image, status or career, ${ }^{\prime 1}$ is at the core of successful team functioning. Google studied successful teams and found diverse personalities and skillsets work together most productively if they incorporate certain team dynamics; chief among these are focusing on shared values and psychological safety. ${ }^{2}$ Times of acute crisis, especially those in which clinicians are working in unfamiliar settings and with new teams, increase the need for psychological safety. During the first wave of the coronavirus disease 2019 (COVID-19) pandemic, many hospitals responded by forming ad hoc teams of non-hospitalist clinicians, including redeployed outpatient physicians and subspecialists. ${ }^{3}$ Because this situation was an acute crisis in which strangers (some new to the field) were suddenly working side by side, it was an excellent example of a moment that required rapid establishment of psychological safety. As subsequent waves of COVID-19 arrive, this will likely occur again. In this perspective, we identify strategies that help to establish psychological safety on medical teams, aiming to increase the effectiveness of teams caring for hospitalized patients, enhance leaders' abilities to improve team function, and allow for delivery of high-quality patient care.

\section{WHY IS PSYCHOLOGICAL SAFETY IMPORTANT?}

Psychological safety creates a nonthreatening team environment in which clinicians can ask questions and seek help with unfamiliar clinical scenarios. When psychological safety is present, the team dynamic encourages interpersonal risk-taking, improves learning, and increases the likelihood that team members will suggest new ideas. ${ }^{4}$ A culture of openness where people feel accepted and respected plays a vital role in helping people thrive in challenging and high-stakes work environments. ${ }^{5}$ In healthcare, team members who do not fear

*Corresponding Author: Lily Rotman Devaraj, MD; Email: LRotman@ mgh.harvard.edu; Telephone: 510-524-1187, 617-626-2000.

Published online first November 18, 2020.

Received: June 21, 2020; Revised: September 24, 2020;

Accepted: September 28, 2020

๑ 2021 Society of Hospital Medicine DOI 10.12788/jhm.3541 punishment for mistakes are more likely to disclose errors. ${ }^{6}$ Psychological safety has been associated with decreased anxiety in stressful situations, thereby freeing learners' mental capacity to explore, innovate, and absorb new information. ${ }^{6}$

\section{STRATEGIES TO IMPLEMENT PSYCHOLOGICAL SAFETY}

Through a thorough literature review, we identified strategies that can increase psychological safety on clinical teams. We focused on strategies applicable to acute crises, like COVID-19, when dynamic teams and uncertainty are rife. These strategies primarily focus on "team leaders," generally the attendings or senior residents, who influence the team's culture.

\section{Discuss Mistakes}

Creating a culture in which openly discussing mistakes is normalized and learning is fostered is especially important for healthcare providers redeployed to COVID-19 wards. Acknowledging errors can be challenging, especially in medicine, because success is often celebrated. ' Creating an environment where discussing mistakes in a nonjudgmental manner is the norm helps people disclose and learn from errors. By modeling fallibility, team leaders can create an environment where learning from mistakes seems less threatening. ${ }^{8}$ Leaders can say, "I may miss something. I encourage all members of the team to share what they know."

\section{Provide Frequent Updates and Seek Feedback} Information and guidelines are changing frequently as we learn more about the novel coronavirus. The barrage of new information and periodic policy changes can be disconcerting. Leaders can dispel some of the team's anxiety by providing a unified message that distills new information into clear and essential updates. ${ }^{9}$ They can reassure the team that updates will be provided frequently, be honest about what is known, and offer some predictability by providing updates at set times via consistent forms of communication during times of crisis. They can show empathy by inquiring about individual worries and responding to concerns about changes that are being made in response to COVID-19. ${ }^{10}$ Leaders should routinely seek feedback. They can ask, "How are things going for you? What can we improve? What should we do differently? How can I make you feel more comfortable or help you learn more effectively?" Inviting input communicates that everyone's opinion is respected and creates a climate where everyone feels comfortable asking questions or respectfully expressing diverging opinions. ${ }^{9}$ 


\section{Foster Creativity and Seek New Ideas}

Curiosity and creativity are associated with better group outcomes. ${ }^{11}$ Curiosity, or the motivation to learn and seek new ideas, improves individual and group dynamics by stimulating better job performance, inspiring leaders to discover more creative solutions, and encouraging employees to develop more trusting relationships, which makes them less likely to stereotype coworkers and patients as they ask questions and learn about others. ${ }^{12}$ People who approach situations with a more creative perspective are less likely to react defensively. Curious people tend to try to learn about and understand different points of view. ${ }^{13}$ For example, if an order is not placed for a patient, a curious team leader might think about why - was there disagreement on the order, confusion on how to place it, or was it inadvertently forgotten?-and be able to help avoid similar scenarios in the future. This ability to see things from another person's point of view helps individuals with diverse clinical, social, and ethnic backgrounds function as a harmonious team. An attitude of curiosity and interest in learning about what each person can contribute based on their unique training and personality will help well-functioning teams form in response to COVID-19. Openness to new ideas allows for more innovative solutions, which are important in times of crisis. To promote curiosity, team members should discuss differences and varied opinions openly. This open dialogue provides individuals opportunities to learn from each other. ${ }^{5}$

\section{Build Connection and Trust}

A culture of trust-the belief that others will act for the good of the team-helps create psychological safety..$^{10}$ Leaders can build trust by making expectations clear, being consistent, being inclusive, and modeling behaviors they wish to encourage. ${ }^{6}$ Predictability reduces anxiety and promotes psychological safety. ${ }^{9}$ Defining goals and expectations helps people relax, ask questions, and focus on learning. ${ }^{14}$ In times of crisis, leaders can tell teams what changes to expect, spell out new priorities, and assign specific tasks to give people a way to contribute..$^{15}$

Activities that create connection also build trust, enhancing the team's sense of psychological safety. Shared experiences foster connections. ${ }^{2}$ Leaders can encourage team bonding by setting aside time to share stories and coping strategies. ${ }^{10}$ Chief residents in the early days of the pandemic found defining social distancing only as physical separation and focusing on emotional bonds helped maintain a sense of community. Debriefing about emotional patient encounters and discussing interesting clinical cases during video calls were ways to implement this strategy. ${ }^{10}$ Team members feel connected to each other and dedicated to their work if they focus on the meaning of the work to them, as well as its impact on society. ${ }^{2}$ This shared belief that what they are doing matters to their community helps bond them. ${ }^{2}$ Currently, the shared experience of treating a novel illness during the COVID-19 pandemic and the common goal of patient well-being unites healthcare providers across the globe. Leaders can create solidarity by emphasizing the shared identity of fighting COVID-19 and reminding teams of the impact of their work. Leaders can say, "Remember, we are here to improve patients' health and form emotional bonds with people and their families." These reminders have been shown to promote psychological safety and connection. ${ }^{16}$

\section{Make Team Members Feel Valued}

As many healthcare providers work harder or in unfamiliar environments during this pandemic, recognition of their efforts by leaders can be especially motivating and meaningful. When individuals on a team feel their work is valued, it helps create a sense of psychological safety. ${ }^{17}$ Employees feel valued when they believe their leaders care, they are in socially supportive environments, and are given resources for professional growth. ${ }^{17}$ Diversity and inclusion are also associated with feeling valued. ${ }^{17}$ During the height of the initial COVID-19 surge at our hospital, the chief of the Department of Medicine regularly sent messages and photographs of trainees and faculty, showing teams coming together during these unprecedented times. This boosted morale and created comradery and is an excellent example of a leader modeling inclusion.

Gratitude strengthens relationships and motivates people, especially when its expression is thoughtful and unique to individuals. ${ }^{18}$ Sincere compliments, acknowledgement of hard work, inclusiveness, and gratitude all contribute to team members feeling at ease and are key to leading, especially in times of crisis. ${ }^{17}$ Leaders can provide motivation by affirming the team's ability to work together. Leaders can say, "I believe in each and every one of your capabilities - and I believe even more so in our joint capabilities. We can do this together." ${ }^{19}$

\section{CONCLUSION}

Psychological safety is a powerful predictor of team performance, increased engagement, and satisfaction. It is critical for creating teams that can deal with uncertainty in high-risk situations, promoting a culture that is safe to acknowledge mistakes and take chances, which is important for optimal team functioning. Crises like the COVID-19 pandemic emphasize the need for psychologically safe team climates to promote learning, safe patient care, and team support. Hospitalists often care for patients when they are at their most vulnerable. Respecting and connecting with patients, through good and efficient teamwork, is important to providing effective care. The strategies suggested in this article strive to help hospitalists create a respectful culture to strengthen relationships with patients and colleagues in order to create an inclusive environment in times of crisis.

Disclosures: The authors have no conflicts of interest, including financial affiliations, to report.

\section{References}

1. Kahn WA. Psychological conditions of personal engagement and disengagement at work. Acad Manage J. 1990;33(4):692-724. https://doi. org/10.5465/256287

2. Rozovsky J. The five keys to a successful Google team. re:Work. Posted November 17, 2015. Accessed October 11, 2020. https://rework.withgoogle. com/blog/five-keys-to-a-successful-google-team/

3. Hettle D, Sutherland K, Miles E, et al. Cross-skilling training to support 
medical redeployment in the COVID-19 pandemic. Future Healthc J. 2020:fhj.2020-0049. https://doi.org/10.7861/fhj.2020-0049

4. Edmondson AC, Lei Z. Psychological safety: the history, renaissance, and future of an interpersonal construct. Annu Rev Organ Psychol Organ Behav 2014;1(1):23-43. https://doi.org/10.1146/annurev-orgpsych-031413-091305

5. Edmondson A. Psychological safety and learning behavior in work teams. Admin Sci Quart.1999;44(2):350-383. https://doi.org/10.2307/2666999

6. Turner S, Harder N. Psychological safe environment: a concept analysis. Clin Simul Nurs. 2018;18:47-55. https://doi.org/10.1016/j.ecns.2018.02.004

7. Jug $R$, Jiang $X S$, Bean $S M$. Giving and receiving effective feedback: a review article and how-to guide. Arch Pathol Lab Med. 2019;143(2):244-250. https:// doi.org/10.5858/arpa.2018-0058-RA

8. Ende J. Feedback in clinical medical education. JAMA. 1983;250(6):777-781. https://doi.org/10.1001/jama.1983.03340060055026

9. Edmondson AC, Woolley AW. Understanding outcomes of organizational learning interventions. In: Easterby-Smith M, Lyles M, eds. Blackwell Handbook of Organizational Learning and Knowledge Management. Blackwell Publishing; 2003.

10. Rakowsky S, Flashner BM, Doolin J, et al. Five questions for residency leadership in the time of COVID-19: reflections of chief medical residents from an internal medicine program. Acad Med. 2020;95(8):1152-1154. https://doi. org/10.1097/ACM.0000000000003419

11. Armstrong K. If you can't beat it, join it: uncertainty and trust in medicine. Ann Intern Med. 2018;168(11):818-819. https://doi.org/10.7326/M18-0445
12. Gino F. The business case for curiosity. Harvard Bus Rev. 2018;96(5):48-57.

13. Kashdan TB, DeWall CN, Pond RS, et al. Curiosity protects against interpersonal aggression: cross-sectional, daily process, and behavioral evidence. $J$ Pers. 2013;81(1):87-102. https://doi.org/10.1111/j.1467-6494.2012.00783.x

14. Epstein RM, Krasner MS. Physician resilience: what it means, why it matters, and how to promote it. Acad Med. 2013;88(3):301-303. https://doi. org/10.1097/ACM.0b013e318280cffO

15. Petriglieri G. The psychology behind effective crisis leadership. Harvard Bus Rev. Published April 22, 2020. Accessed October 11, 2020. https://hbr. org/2020/04/the-psychology-behind-effective-crisis-leadership

16. Edmondson A. Building a psychologically safe workplace: TEDx Talk. May 4, 2014. Accessed October 11, 2020. https://youtube.com/watch?v=LhoLuuiggX8

17. Simpkin AL, Chang Y, Yu L, Campbell EG, Armstrong K, Walensky RP. Assessment of job satisfaction and feeling valued in academic medicine. JAMA Intern Med. 2019;179(7):992-994. https://doi.org/10.1001/jamainternmed.2019.0377

18. Nawaz S. In times of crisis, a little thanks goes a long way. Harvard Bus Rev. Published May 22, 2020. Accessed October 11, 2020. https://hbr. org/2020/05/in-times-of-crisis-a-little-thanks-goes-a-long-way

19. Knight R. How to talk to your team when the future is uncertain. Harvard Bus Rev. Published April 20, 2020. Accessed October 11, 2020. https://hbr. org/2020/04/how-to-talk-to-your-team-when-the-future-is-uncertain 